\title{
Anticorrelation between the Bulk Speed and the Electron Temperature in the Pristine Solar Wind: First Results from the Parker Solar Probe and Comparison with Helios
}

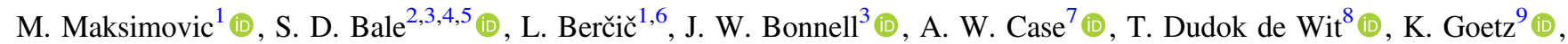

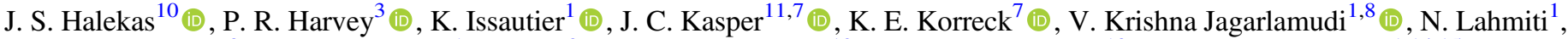

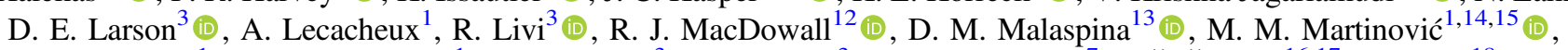

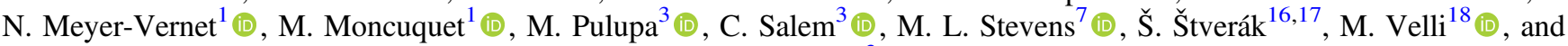
P. L. Whittlesey ${ }^{3}$ (1)

${ }^{1}$ LESIA, Observatoire de Paris, Université PSL, CNRS, Sorbonne Université, Université de Paris, 5 place Jules Janssen, F-92195 Meudon, France; milan.maksimovic@obspm.fr

${ }^{2}$ Physics Department, University of California, Berkeley, CA 94720-7300, USA

${ }^{3}$ Space Sciences Laboratory, University of California, Berkeley, CA 94720-7450, USA

${ }^{4}$ The Blackett Laboratory, Imperial College London, London, SW7 2AZ, UK

${ }^{5}$ School of Physics and Astronomy, Queen Mary University of London, London E1 4NS, UK

${ }^{6}$ Physics and Astronomy Department, University of Florence, Via Giovanni Sansone 1, I-50019 Sesto Fiorentino, Italy ${ }^{7}$ Smithsonian Astrophysical Observatory, Cambridge, MA 02138, USA

${ }^{8}$ LPC2E, CNRS and University of Orléans, Orléans, France

${ }^{9}$ School of Physics and Astronomy, University of Minnesota, Minneapolis, MN 55455, USA

${ }^{10}$ Department of Physics and Astronomy, University of Iowa, IA 52242, USA

${ }^{11}$ Climate and Space Sciences and Engineering, University of Michigan, Ann Arbor, MI 48109, USA

12 Solar System Exploration Division, NASA/Goddard Space Flight Center, Greenbelt, MD 20771, USA

${ }^{13}$ Laboratory for Atmospheric and Space Physics, University of Colorado, Boulder, CO 80303, USA

${ }_{15}^{14}$ Lunar and Planetary Laboratory, University of Arizona, Tucson, AZ 85719, USA

${ }^{15}$ Department of Astronomy, Faculty of Mathematics, University of Belgrade, Serbia

${ }^{16}$ Astronomical Institute, Czech Academy of Sciences, CZ-14100 Prague, Czech Republic

${ }^{17}$ Institute of Atmospheric Physics, Czech Academy of Sciences, CZ-14100 Prague, Czech Republic

${ }^{18}$ Department of Earth, Planetary \& Space Sciences, University of California, Los Angeles, CA 90095, USA

Received 2019 September 29; revised 2019 December 12; accepted 2019 December 12; published 2020 February 3

\begin{abstract}
We discuss the solar wind electron temperatures $T_{e}$ as measured in the nascent solar wind by Parker Solar Probe during its first perihelion pass. The measurements have been obtained by fitting the high-frequency part of quasithermal noise spectra recorded by the Radio Frequency Spectrometer. In addition we compare these measurements with those obtained by the electrostatic analyzer discussed in Halekas et al. These first electron observations show an anticorrelation between $T_{e}$ and the wind bulk speed $V$ : this anticorrelation is most likely the remnant of the wellknown mapping observed at 1 au and beyond between the fast wind and its coronal hole sources, where electrons are observed to be cooler than in the quiet corona. We also revisit Helios electron temperature measurements and show, for the first time, that an in situ $\left(T_{e}, V\right)$ anticorrelation is well observed at 0.3 au but disappears as the wind expands, evolves, and mixes with different electron temperature gradients for different wind speeds.
\end{abstract}

Unified Astronomy Thesaurus concepts: Solar wind (1534)

\section{Introduction}

In thermally driven solar wind models, for which the coronal thermal energy is converted into kinetic solar wind bulk energy, the final asymptotic speed is a function of the initial coronal temperature (Parker 1958). The hotter the corona, the faster the wind. While this property seems to be verified from remote sensing spectroscopic observations of the corona for minor ions and hydrogen, it does not appear to be correct for the electrons.

Although measuring the coronal proton temperature by means of ultraviolet coronograph spectrometers is a complex task, it seems well established now that the fast solar wind originates from coronal holes where the hydrogen kinetic temperatures are possibly as large as 4-6 million $\mathrm{K}$ (Kohl et al. 1996; Cranmer 2002). In addition, the direct correlation between proton temperature and wind speed seems to persist throughout the heliosphere as the well-known correlation between the in situ proton temperature $T_{p}$ and the bulk speed $V$, amply described in the literature (Lopez \& Freeman 1986;
Arya \& Freeman 1991; Totten et al. 1995; Matthaeus et al. 2006), and references therein.

As far as electrons are concerned, their temperature in coronal holes is observed to be lower than that in the quiet corona (David et al. 1998; Wilhelm et al. 1998; Doschek et al. 2001; Cranmer 2002). In addition the so-called freezing-in temperature of solar wind minor ions, a proxy for the electron temperature at the coronal source obtained from in situ heliospheric measurements of different heavy ion charge states, exhibits a clear anticorrelation with the local solar wind bulk speed V (Geiss et al. 1995; Ko et al. 1997; Gloeckler et al. 2003; von Steiger \& Zurbuchen 2011). This latter anticorrelation has also been inferred by Marsch et al. (1989), who used Helios electron data. These authors extrapolated back to the Sun the electron temperature using power-law variations of the latter as a function of the solar wind speed.

In this paper we present observations of the solar wind electron temperatures $T_{e}$ measured by the Parker Solar Probe (PSP; Fox et al. 2016) during its first perihelion pass in what we may consider to be a nascent, or pristine, solar wind. These 
measurements have been obtained by using the quasi-thermal noise spectroscopy technique on the one hand and the spacecraft electrostatic analyzer on the other. The measurements reveal a solar wind in the region around 35 solar radii exhibiting a clear and direct anticorrelation between $T_{e}$ and $V$, as also reported in the companion paper by Halekas et al. (2020). Building on this finding, we revisit Helios electron temperature measurements taken at 0.29 au and beyond, using an analysis technique similar to the one of Marsch et al. (1989). We show, for the first time directly, that the $\left(T_{e}, V\right)$ anticorrelation is well established also at 0.3 au but is cleared gradually as the wind expands (and mixes) with different electron temperature gradients for different wind speeds.

In Section 2 we describe measurements of the solar wind electrons on PSP. We detail how electron temperatures are retrieved from the high-frequency part of the quasi-thermal noise spectra obtained by the electric field antennae. In Section 3 we first describe the published Helios data sets, which we proceed to reanalyze. Finally, in Section 4 we discuss the implications of our results for the dynamical processes underlying solar wind acceleration and evolution.

\section{PSP Electron Temperature Measurements}

The solar wind electron velocity distribution functions (VDFs), observed at distances of 0.29 au from the Sun and beyond, systematically exhibit three different recognizable components: a thermal core and a suprathermal halo, always present at all pitch angles, and a sharply magnetic field-aligned strahl that usually moves in the antisunward direction (Feldman et al. 1975; Rosenbauer et al. 1977; Pilipp et al. 1987; Maksimovic et al. 1997). Whereas the effects of Coulomb collisions may explain the relative isotropy of the core population, the origin of the halo and core populations and their interplay during the wind expansion are currently strongly debated (Maksimovic et al. 2005; Štverák et al. 2009; Berčič et al. 2019; Horaites et al. 2019). Are the electron VDFs already non-thermal in the corona as proposed by Scudder (1992a, 1992b) or does this characteristic arise from the expansion of a weakly collisional Maxwellian atmosphere? One of the key science objectives, among others, of measuring the properties of solar wind electrons with PSP, is to understand the origin and evolution of the electron distribution functions and their dynamical role in solar wind acceleration.

In this paper we use data from both the FIELDS (Bale et al. 2016)) and the Solar Wind Electrons Alphas and Protons (SWEAP; Kasper et al. 2016) instruments on PSP. Solar wind electrons may be diagnosed accurately, both by analyzing the quasi-thermal noise (QTN) spectra (Meyer-Vernet et al. 2017), recorded by the RFS low-frequency receiver (Pulupa et al. 2017) using the FIELDS electric antennas (Bale et al. 2016), or by direct detection of their VDFs with the Solar Probe Analyzer (SPAN) on board SWEAP (P. Whittlesey et al. 2020, in preparation). In the next subsection we detail the way in which the total electron temperature may be retrieved from the highfrequency part of the RFS spectra using QTN analysis, and in the subsequent one we present some of the early SWEAP electron measurements, which will be shown to be broadly consistent with those obtained via QTN.

\subsection{Quasi-thermal Noise Measurements Using the High- frequency Part of the RFS Spectra}

When immersed in a space plasma, an antenna will measure the electrostatic fluctuations induced by the quasi-thermal motion of the ambient electric charges which surround it. The theory of antenna-plasma coupling in space environments out of thermal equilibrium and its use for actual measurements is now well established (Meyer-Vernet 1979; Meyer-Vernet \& Perche 1989; Meyer-Vernet et al. 2017). The so-called QTN spectroscopy has been applied successfully to several past missions operating in the solar wind such as ISSE 3 (MeyerVernet 1979; Couturier et al. 1981), Ulysses (Maksimovic et al. 1995; Issautier et al. 1999), Wind (Maksimovic et al. 1998), or STEREO (Martinović et al. 2016). It has been implemented on the FIELDS instrument to provide accurate measurements of the electron density and temperature of the outer corona down to 10 solar radii (Bale et al. 2016).

The first results of the QTN measurements on PSP are described in detail in this special issue by Moncuquet et al. (2020). These authors present a method that yields the total electron density $N_{e}$, the core temperature $T_{c}$, and a suprathermal temperature. Note that in this study, this latter suprathermal temperature is actually the total contribution of the halo + strahl thermal pressure.

As a complementary method, we use, in the present paper, the high-frequency part of the QTN spectra recorded by RFS, above the plasma peak. For this frequency range, the contributions of the proton Doppler-shifted thermal noise and the electron shot noise, including the current-biasing of the antennas, which is performed for DC electric fields measurements, are negligible (Meyer-Vernet et al. 2017). Moreover our QTN analysis is mostly dependent on the electron total thermal pressure. For frequencies $f$ satisfying $f \gg f_{p} L_{\mathrm{D}} / L$ where $f_{p}$ and $L_{\mathrm{D}}$ are the local plasma frequency and Debye length, respectively, and $L$ is the physical length of one arm of the dipole, the QTN is proportional to $N_{e} T_{e} / f^{3}$ (Meyer-Vernet \& Perche 1989).

Another important contribution that needs to be considered in our analysis and which can be clearly observed by the RFS radio receiver is the galactic radio background (Novaco \& Brown 1978; Cane 1979). This background radiation is relatively constant and covers a $4 \pi$ solid angle roughly isotropically. Note that modulations of the galactic background as a function of the observed solid angle are less than $20 \%$ in the frequency range of consideration (Manning \& Dulk 2001). To obtain the pure QTN spectrum at high frequency, we have to properly subtract the radio galactic background from the total signal.

Figure 1 displays a typical power spectral density (PSD) RFS spectrum between $100 \mathrm{kHz}$ and $10 \mathrm{MHz}$ of the FIELDS $|V 1-V 2|$ antenna dipole. These observations, represented by black diamonds, have been obtained by merging the RFS/HFR and RFS/LFR spectra in one. We did the same for all the data of the first perihelion used here. The dotted horizontal line represents the RFS pre-deployment internal noise, which is $V_{\text {noise }}^{2} \sim 2.2 \times 10^{-17} \mathrm{~V}^{2} \mathrm{~Hz}^{-1}$ in the considered frequency range (Pulupa et al. 2020). The black dashed line on Figure 1 represents the RFS PSD $V_{\text {galaxy }}^{2}$ corresponding to the Novaco \& Brown (1978) radio galaxy model that we have computed using the detailed method described by Zaslavsky et al. (2011). More precisely we have used an RFS spectrum measured when PSP was close to $1 \mathrm{au}$. At this distance the plasma peak (around 20 


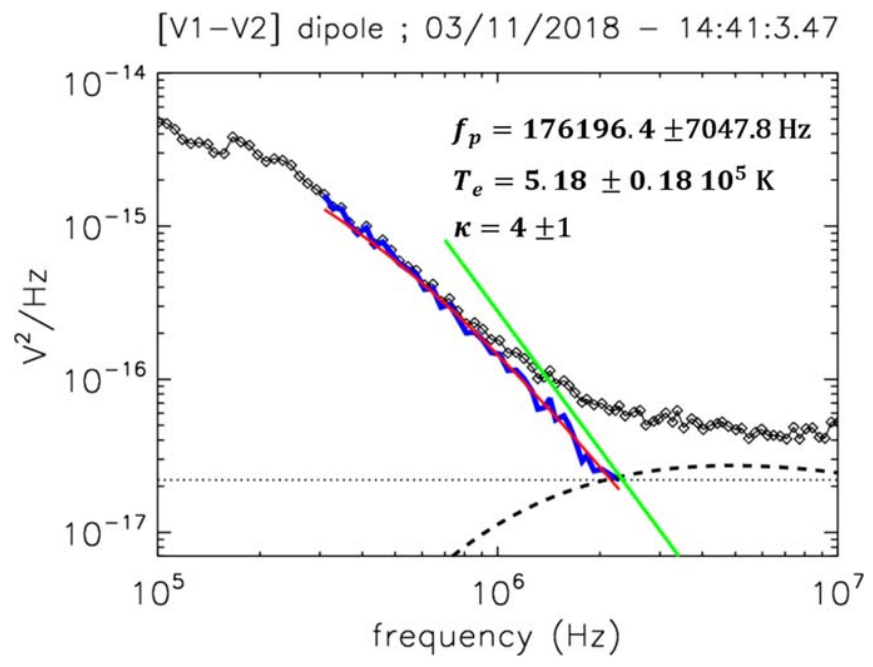

Figure 1. Example of a power spectrum density RFS spectrum measured between $100 \mathrm{kHz}$ and $10 \mathrm{MHz}$ by the FIELDS $|V 1-V 2|$ antenna dipole (back diamonds). The dotted horizontal line represents the RFS pre-deployment internal noise of $\sim 2.2 \times 10^{-17} \mathrm{~V}^{2} \mathrm{~Hz}^{-1}$. The black dashed line represent the radio galaxy model. The blue and red lines represent the data and the model respectively, which we used for the QTN fitting method described in the text. The green straight line represents an $f^{-3}$ variation that the QTN spectrum should follow for $f \gg f_{p} L_{\mathrm{D}} / L$

$\mathrm{kHz}$ ) is much lower than the typical $f_{p}$ for the current study. The corresponding QTN spectrum is therefore negligible in the $1-10 \mathrm{MHz}$ range at $1 \mathrm{au}$. Then taking the observed value of the RFS PSD in the range $2-3 \mathrm{MHz}$ and subtracting the above defined receiver noise one obtains a value of $\sim 2.3 \times 10^{-17} \mathrm{~V}^{2} \mathrm{~Hz}^{-1}$ for the galaxy PSD in this frequency range. Finally, using the Novaco \& Brown (1978) model, which yields a radio brightness of $1.1 \times 10^{-20} \mathrm{~W} \mathrm{~m}^{-2} \mathrm{~Hz}^{-1} \mathrm{sr}^{-1}$ for the corresponding range and applying formula (13) from Zaslavsky et al. (2011) we obtain a reduced effective length of $\Gamma L_{\text {eff }}=1.17$, which we can then use for computing $V_{\text {galaxy }}^{2}(f)$ on Figure 1.

We can now fit the RFS QTN spectra using the following procedure for each individual RFS $|V 1-V 2|$ spectrum. We first select all the data frequency points verifying $V_{\text {obs }}^{2}(f)>3 \times V_{\text {noise }}^{2}$ in the frequency range $300 \mathrm{kHz}-$ $20 \mathrm{MHz}$. The $300 \mathrm{kHz}$ lower-frequency limit has been chosen to be well above $f_{p}$ so that, as described previously, the proton QTN and the shot noise can be neglected. Then we remove the receiver noise and the galaxy spectrum in order to define the observed QTN spectra $V_{\text {obs-QTN }}^{2}(f)=V_{\text {obs }}^{2}(f)-V_{\text {noise }}^{2}-V_{\text {galaxy }}^{2}(f)$ to which we can apply the QTN spectroscopy. The blue curve on Figure 1 represents $V_{\text {obs-QTN }}^{2}(f)$ for the considered spectrum. In this particular case $V_{\text {obs-QTN }}^{2}(f)$ is only visible up to $2.3 \mathrm{MHz}$. Above this frequency, it is lower than the sum of the receiver noise and the galaxy spectrum and thus cannot be measured. The displayed $V_{\text {obs-QTN }}^{2}(f)$ spectrum is defined on 38 frequency points. The spectra we fit in this study are defined on a number of frequency points, which is usually comprised between $\sim 10$ and $\sim 50$ and are never defined on frequencies over $3.5 \mathrm{MHz}$, because of the galactic background. The final step now consists of fitting $V_{\text {obs-QTN }}^{2}(f)$ using a full and comprehensive QTN model with Lorentzian VDFs for the electrons (Chateau \& Meyer-Vernet 1989; Zouganelis 2008; Le Chat et al. 2011). This kind of model is well adapted for the high-frequency part of the spectra, which is mostly sensitive to the total electron

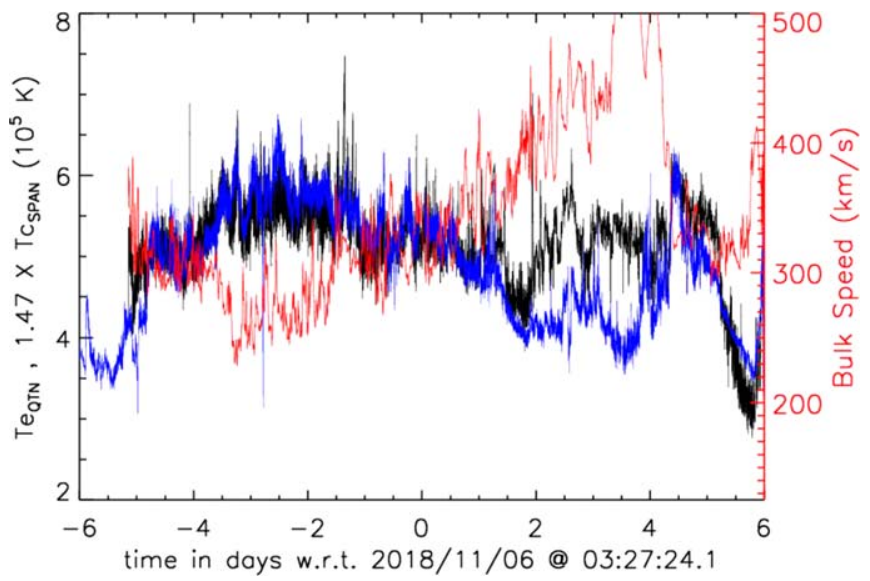

Figure 2. Parker Solar Probe electron temperatures $T_{e, \mathrm{QTN}}$ (in black) and $1.47 \times T_{c \text {,SPAN }}$ (in blue), for 12 days around the date of the first perihelion. The solar wind bulk speed $V$ is displayed in red. An anticorrelation between $V$ and both $T_{c, \text { SPAN }}$ and $T_{e, \mathrm{QTN}}$ is clearly visible across the time interval.

thermal pressure and less to the precise shape of the model VDFs. The red curve on Figure 1 represents the theoretical Lorentzian QTN spectrum, which fits the best $V_{\text {obs-QTN }}^{2}$. Note that for our fitting procedure we only have two free parameters, which are the total temperature $T_{e}$ and the index $\kappa$ of the electron VDFs (see Chateau \& Meyer-Vernet 1991; Zouganelis 2008; Le Chat et al. 2011). The plasma frequency for each fitted spectrum is set to be the one obtained by the peak tracking method used by Moncuquet et al. (2020) with an uncertainty of $4 \%$, which is the standard frequency resolution of the RFS. As for $T_{e}$ and the index $\kappa$, the uncertainties indicated on the figure correspond to the resolution of the model grid that was used for our fitting. Finally, the green straight line on Figure 1 represents an $f^{-3}$ variation that compares well to $V_{\text {obs-QTN }}^{2}(f \geqslant 1.5 \mathrm{MHz})$.

The QTN temperatures $T_{e \text {,QTN }}$ are represented as the black line on Figure 2 for 12 days around the date of the first perihelion. $T_{e, \mathrm{QTN}}$ is ranging between 3 and $6 \times 10^{5} \mathrm{~K}$ in good agreement with an extrapolation of typical 1 au electron temperatures in the range $1-2 \times 10^{5} \mathrm{~K}$ and with a typical radial gradient of $T_{e} \propto R^{-(0.6-0.8)}$ observed in the inner heliosphere (Maksimovic et al. 2000). Note that we have displayed in Figure 2 only 50\% of the data for which the fittings yield a $\chi^{2}$, which is lower than the median of all $\chi^{2}$.

\subsection{SPAN Electron Measurements}

The SPAN instrument is composed of two electron sensors on the ram and anti-ram faces of the spacecraft. They together measure the majority of the three-dimensional electron VDF as explained in detail by P. Whittlesey et al. (2020, in preparation). The first SPAN observations obtained during the first two $P S P$ perihelia show that there is a large strahl/halo density ratio at $35 R_{s}$ (Halekas et al. 2020). This is in good agreement with the expectations from Maksimovic et al. (2005) and Štverák et al. (2009).

In this paper we are using data provided by Halekas et al. (2020), who have developed a robust fitting procedure to retrieve the electron core temperature $T_{c, \text { SPAN }}$. This temperature is displayed in Figure 2 as the blue line. In order to provide a better visual comparison between $T_{e, \mathrm{QTN}}$ and $T_{c, \text { SPAN }}$ we have multiplied arbitrarily the latter by 1.47 , which is the median value of $T_{e, \mathrm{QTN}} / T_{c, \mathrm{SPAN}}$ in the considered time interval. This 
factor measures the actual contribution of the suprathermal population (halo and strahl) to the electron thermal pressure. Overall, the agreement between $T_{e, \mathrm{QTN}}$ and $T_{c, \mathrm{SPAN}}$ is quite good. The linear Pearson correlation between the two data sets is equal to 0.57 for the whole time interval of Figure 2. One should note, however, that there is a discrepancy between the two temperatures during a time interval ranging between 2 and 4 days after the perihelion, which occurred on 2018 November 6 at 03:27. The reason for this discrepancy is still under investigation and is outside the scope of the present paper.

On Figure 2 we have also displayed the solar wind bulk speed $V$, in red, as measured by the SWEAP Faraday cup instrument (Kasper et al. 2016). A clear anticorrelation between $V$ and both $T_{c, \text { SPAN }}$ and $T_{e, \mathrm{QTN}}$ is visible across the time interval. This anticorrelation, which is more pronounced between $V$ and $T_{c, \text { SPAN }}$ (linear Pearson correlation of -0.59) than between $V$ and $T_{e, \mathrm{QTN}}$ (correlation of -0.20 ), despite the good correlation between the two temperatures, has also been reported by Halekas et al. (2020). We now explore this property by revisiting the Helios data.

\section{Helios Observations Revisited}

\subsection{Helios Data Sets}

In this paper we use three different data sets from the ions and electron electrostatic analyzers on board the Helios 1 and 2 spacecraft (Schwenn et al. 1975), which performed in-ecliptic measurements in the heliocentric distance range between 0.3 and $1 \mathrm{au}$. The first data set contains $\sim 1,877,000$ measurements of proton density $N_{p}$, temperature $T_{p}$ and bulk speed $V$ measurements. This constitutes the original Helios plasma data set available at ftp://cdaweb.gsfc.nasa.gov/pub/data/helios/ helios $1 /$ merged $/$.

The second and third data sets contain electron density $N_{e}$ and temperature $T_{e}$ measurements obtained by fitting the velocity distributions recorded by the I2 electron analyzer (Rosenbauer et al. 1977; Pilipp et al. 1987). This analyzer was designed to measure only 2D distribution functions with an aperture pointing in the ecliptic plane.

The second data set we use has been obtained by Štverák et al. (2009). These authors used only those measurements for which the magnetic field vector is close enough to the ecliptic plane, that is, when $B_{z} /|B|<0.1$. This way they restricted their analysis to the good measurements of the VDFs in the $\left(v_{\perp}, v_{\|}\right)$ plane, where the directions $\perp$ and $\|$ are with respect to the local magnetic field vector. This data set contains $\sim 66000$ measurements of $N_{e}$ and $T$.

Finally, the last data set we used in this work is obtained in a similar way to that of Štverák et al. (2009), but using a slightly different model to describe the velocity distribution of electrons. Details on the data processing can be found in Section 3.2 of Berčič et al. (2019). This last data set is not limited to the times when the magnetic field lies within the 2D field of view of the electron instrument and therefore only provides good estimations of the perpendicular part of the electron temperature. On the positive side, it gives better statistics, with approximately three times as many point as the Štverák et al. (2009) data set.

\subsection{Analysis}

If one displays, for the full Helios data set, the solar wind bulk speed as a function of the heliocentric distance one can

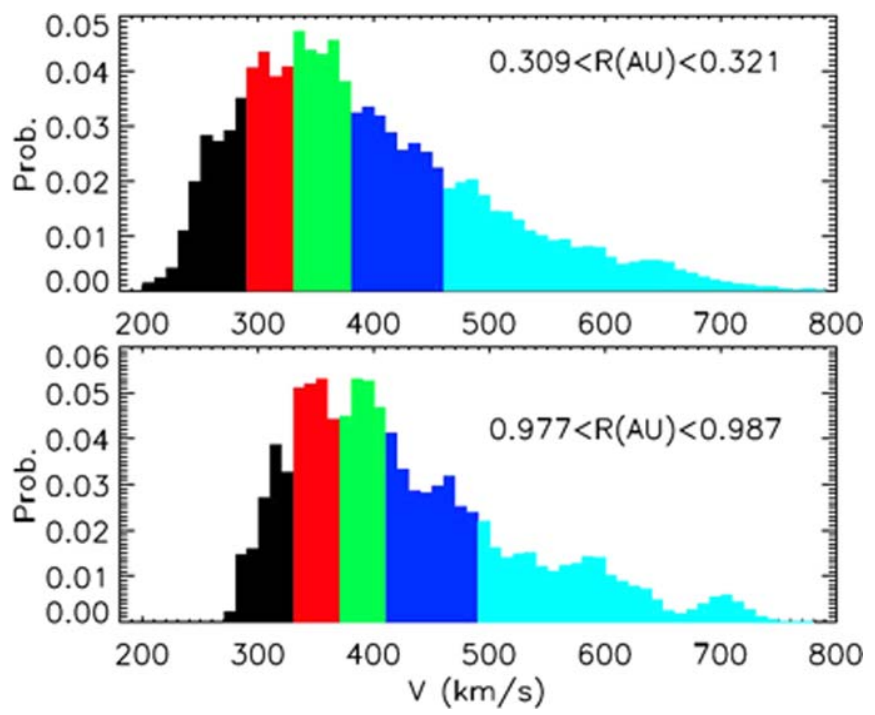

Figure 3. Illustration of the way we define our wind families (see the text for more details).

note that, while the fastest solar wind flows are bounded by a constant value of about $800 \mathrm{~km} \mathrm{~s}^{-1}$, the slowest solar wind streams increase with radial distance between 0.3 and 1 au.

In order to properly quantify this actual acceleration of the slow solar wind we use a procedure illustrated by Figure 3 . We first split the data into 20 heliocentric distance bins. For statistically representative radial coverage, we define these radial bins so that they each contain an equal number of data points, which is $1,877,000 / 20 \sim 93,800$. In each of the radial bins we compute the median value of the heliocentric distance and assign it to the radial bin. We then make the assumption that the lowest quintile of the speed data distribution in the first radial bin at $\sim 0.3 \mathrm{au}$ (black histogram bins on Figure 3) contains the same solar wind streams as the lowest quintile of the data in the following radial bin and so on until the last one at $\sim 0.98$ au. We proceed the same way for the second quintile of the bulk speeds and so on. In this manner we separate the solar wind into five wind families that we display using five color codes (black, red, green, blue, and cyan in order of increasing speed values) throughout the paper. For each of these families we compute the median bulk speeds of the five quintiles in each of the 20 radial bins. This leads to $20\left(R_{i}, V_{i}\right)$ data points, which we display in the upper panel of Figure 4. We proceed the same way with the proton density $\left(R_{i}, N_{p i}\right)$ and total temperature $\left(R_{i}, T_{p i}\right)$ data.

In the upper panel of Figure 4 it can be clearly seen that while the bulk speed of the fastest solar wind (cyan) is approximately constant, the bulk speed for the slowest wind (black) is clearly increasing. In order to quantify this apparent increase, we have performed a linear fit in the form $V(R)=A \times R_{\mathrm{AU}}+V_{0}$ for each of the five wind families. The lower panel of Figure 4 displays variation of the parameters $A$ as a function of $V_{0}$. This $V_{0}$ fitting parameter is used in the following in order to identify the wind families. Except for the fastest wind $\left(V_{0} \approx 600 \mathrm{~km} \mathrm{~s}^{-1}\right)$, all the wind families with $V_{0}<500 \mathrm{~km} \mathrm{~s}^{-1}$ exhibit an increase with radial distance, which is more important as $V_{0}$ decreases. The slowest wind family (black dots on the upper panel of Figure 4) with a $V_{0} \approx 250 \mathrm{~km} \mathrm{~s}^{-1}$ exhibits an increase of speed up to $\approx 90$ $\mathrm{km} \mathrm{s}^{-1}$ per au. 


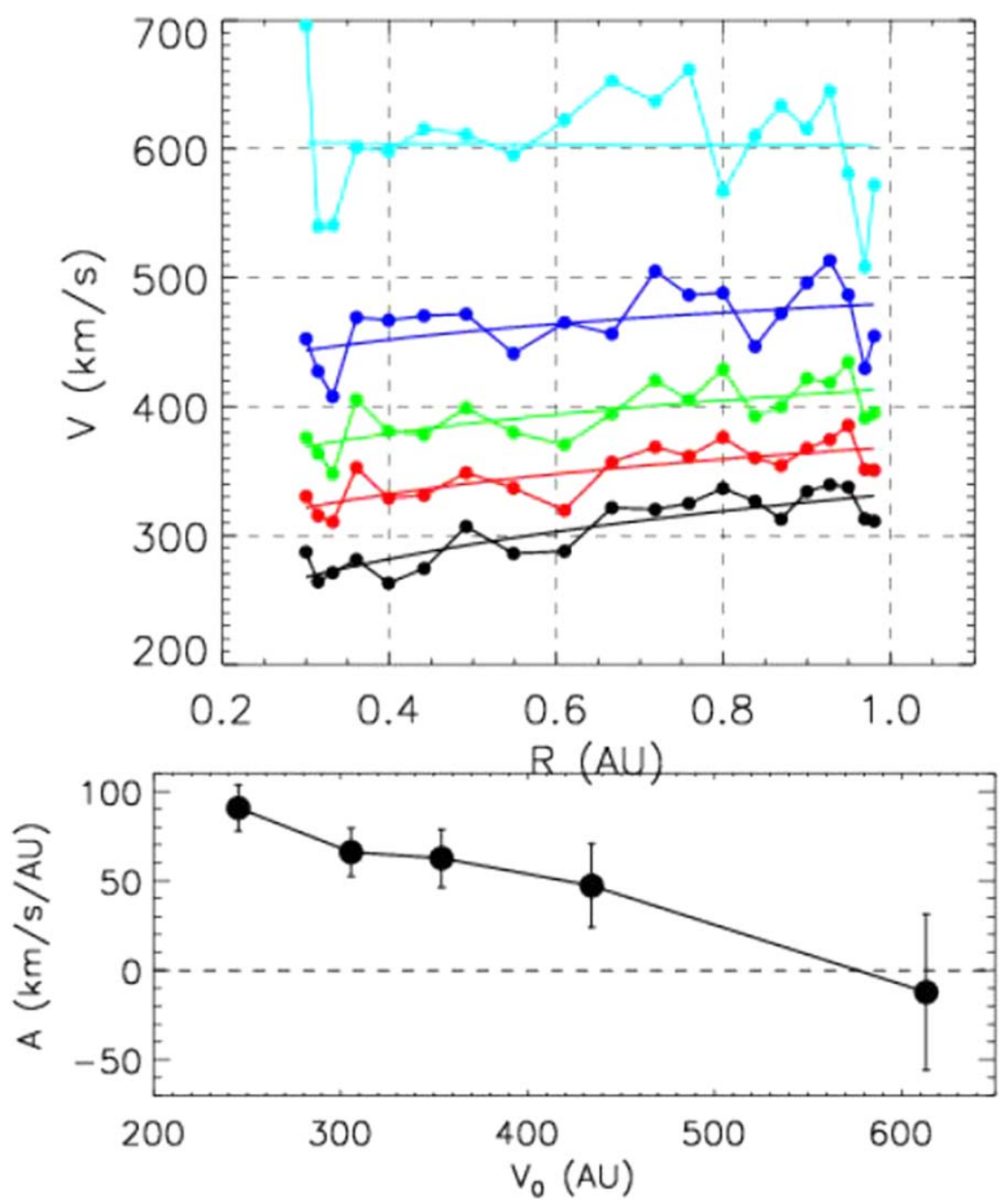

Figure 4. Upper panel: median solar wind bulk speeds as a function of the heliocentric distance for the five wind families. Lower panel: variation of the parameters $A$ as a function of $V_{0}$ from the linear fit $V(R)=A \times R_{\mathrm{AU}}+V_{0}$ for each of the five wind families.

In Figure 5 we display from top to bottom the proton densities $\left(R_{i}, N_{p i}\right)$, proton temperatures $\left(R_{i}, T_{p i}\right)$, and the electron temperatures $\left(R_{i}, T_{e i}\right)$ from the (Štverák et al. 2009) data set for the five wind families. Note that since there are less data points in the electron data set than in the proton one, we have defined 10 radial bins for the latter case instead of 20 . The well-known correlations between the proton bulk speed, density, and temperature can clearly be seen in Figure 5. The fastest is the solar wind (cyan dots), the largest is its proton temperature and the less dense wind. In order to better visualize these correlations, we fit the wind families' proton temperatures with power laws of the form $T_{p}=T_{p 0} \times R_{\mathrm{AU}}^{\alpha_{p}}$. The resulting fits are displayed in Figure 5 by full lines with the same colors used for the wind families. We do the same for the electron temperatures.

We display the outputs of the above power-law fittings in Figure 6. One au temperatures $T_{p 0}$ and $T_{e 0}$ (upper panel) and power-law indexes $\alpha_{p}$ and $\alpha_{e}$ (lower panel) are displayed as a function of $V_{0}$ for the protons (in red) and the electrons (in black), respectively. We can see in this figure one of the major results of this article. While the proton temperature gradients are very similar for the five families, with a average power index of $\approx-0.9$, this is not the case for the electron gradients. The temperature gradients for the electrons are quite different, with a clear tendency for the slow wind electron to cool down more steeply $\left(\alpha_{e} \approx-0.8\right)$ than the fast wind ones $\left(\alpha_{e} \approx-0.3\right)$. This result is consistent with those obtained by Ṡtverák et al. (2015), who show that for the slow wind the electron temperature power-law radial dependence is $T_{e} \propto r^{-0.59}$, while it is $T_{e} \propto r^{-0.31}$ for the fast wind. It is also consistent with the outcome of the study by Marsch et al. (1989), who applied a slightly different technique for binning the data with respect to the bulk speed. Marsch et al. (1989) actually used the same interval limits for the speed bins at all radial distances. This technique, although similar to ours, does not account for the acceleration of the solar wind, especially for the slowest one. The red triangles connected by a dashed line in the lower panel of Figure 6 represent the $\alpha_{e}$ power-law index as a function of $V$ from Marsch et al. (1989). Note also that the proton temperature power-law indexes we obtain are very similar to those retrieved by Totten et al. (1995), who used the same technique as Marsch et al. (1989) for binning the data by bulk speed. The black triangles connected by a dashed line in the lower panel of Figure 6 represent the $\alpha_{p}$ power-law index as a function of $V$ from Totten et al. (1995). Considering the values of $T_{p 0}$ and $T_{e 0}$, which are the proton and electron modeled temperatures at $1 \mathrm{au}$, we retrieve the well-known and established correlation between the proton temperature and 

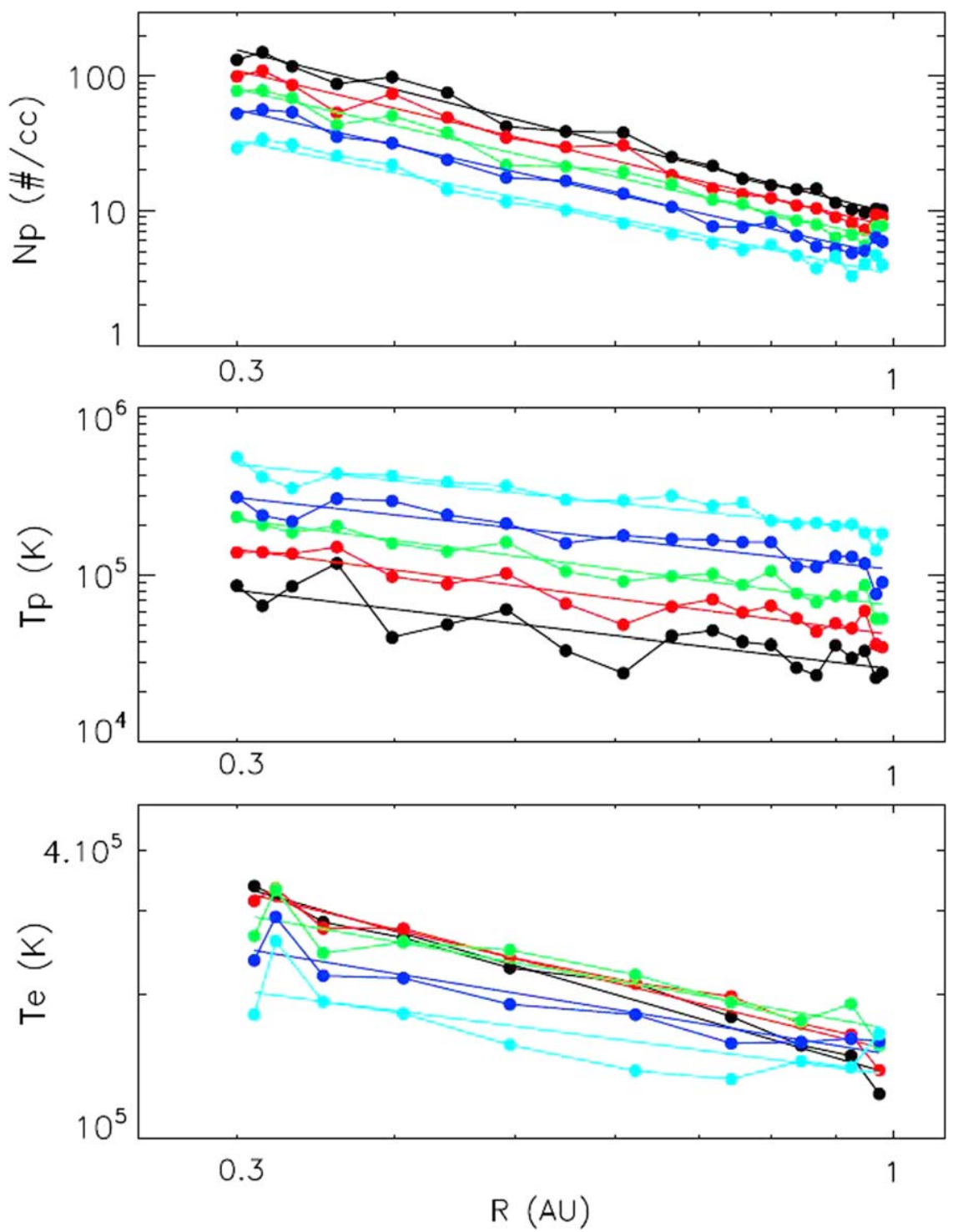

Figure 5. From top to bottom: proton density, proton temperature, and electron temperature as a function of the radial distance, measured by Helios. The colored lines show the binned data for our wind families (see the text for more explanation).

the wind bulk speed and the lack of such a correlation for the electrons.

We now explore how these correlations evolve with radial distance. First, we downsample the proton data set to times when the electron measurements in the Štverák et al. 2009) data set were performed. We thus present correlations on the same statistical basis for protons and electrons. As done for the latter previously, we now compute the median values $\left(V_{i}, T_{p i}\right)$ on 10 radial bins $R_{i}$ instead of 20 . The upper panel of Figure 7 displays the proton temperature as a function of the bulk speed for all the points (light dots) and the medians (thick dots) for the first (black), the sixth (red), and the tenth (blue) radial bins. These three radials bins correspond to the following heliocentric distance ranges, respectively: $0.29<R_{\mathrm{AU}}<0.31$, $0.55<R_{\mathrm{AU}}<0.69,0.95<R_{\mathrm{AU}}<0.98$. Because the $T_{p}$ gradients are similar for all the wind families the 1 au $\left(V, T_{p}\right)$ correlation is maintained at all radial distances. This is true both for the medians and for all the data points within a radial bin. This can be seen quantitatively in Table 1 , where we have reported the linear Pearson correlation coefficients between $V$ and $T_{p}$ for the radial bin data defined above. While the correlation coefficient between the proton temperature and speed is equal to 0.75 close to $1 \mathrm{au}$, it falls down to 0.55 close to $0.3 \mathrm{au}$, but still remains.

For the electrons the tendency is the opposite, as one can see in the lower panel of Figure 7. There is a strong $\left(V, T_{e}\right)$ anticorrelation at 0.3 , with a correlation coefficient of -0.70 . This property is most likely the remnant of the coronal electron temperature mapping. However, as the $T_{e}$ gradients are quiet different for the different wind families, the pristine $\left(V, T_{e}\right)$ anticorrelation is cleared as the wind expands. At 1 au the anticorrelation has completely disappeared on the Helios data, with a very low $\left(V, T_{e}\right)$ correlation coefficient of 0.31 . The same trend is also observed using the Berčič et al. (2019) data set, for which we have computed the correlations between $V$ and $T_{e \perp}$, the total electron temperature in the perpendicular direction to the magnetic field.

Finally, in Figure 8 we have combined the Helios electron median temperatures displayed in Figure 7 with those measured by $P S P$ and discussed in Section 2. For $P S P$ we have only selected data in the radial range $35.7 R_{S}<R<45 R_{S}$ and binned them on quartiles according to their bulk speeds. $T_{e, \mathrm{QTN}}$ 

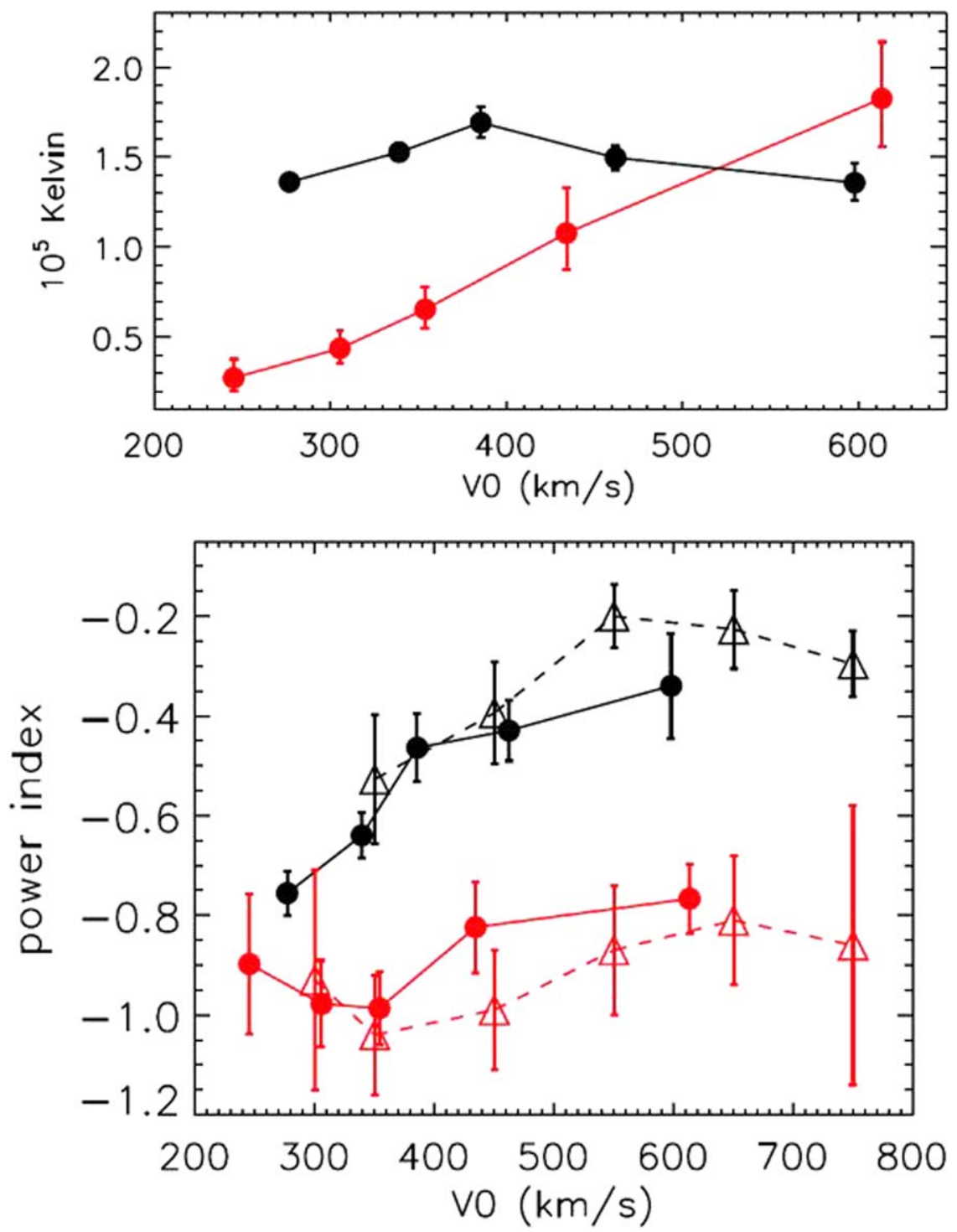

Figure 6. Outcome of the power-law modeling of the form $T=T_{0} \times R_{\mathrm{AU}}^{\alpha}$ : one au temperatures $T_{p 0}$ and $T_{e 0}$ (upper panel) and power-law indexes $\alpha_{p}$ and $\alpha_{e}$ (lower panel) are displayed as a function of $V_{0}$ for the protons (in red) and the electrons (in black; see the text for more details). While the proton temperature gradients are very similar for the five wind speed families, with an average power index of $\approx-0.9$, this is not the case for the electrons that exhibit a clear tendency for the slow wind to cool down more steeply $\left(\alpha_{e} \approx-0.8\right)$ than the fast one $\left(\alpha_{e} \approx-0.3\right)$.

is displayed in magenta, while $1.47 \times T_{c, \text { SPAN }}$ is displayed in orange. Note that the limit of the slow wind observed by Helios at $66 R_{s}$ is shifted to even slower wind speeds at $35 R_{s}$, illustrating once more the strong wind acceleration that occurs for the slow wind streams. Another very interesting curve is displayed in green on Figure 8. It corresponds to the measurements of the total electron temperatures obtained with the 3DP electrostatic analyzer on board Wind (Lin et al. 1995). For this curve we have accumulated four years of Wind data (1995-1998) and displayed the medians of $T_{e}$ for 8 bins of bulk speed $V$. The Wind $T_{e}$ variations as a function of $V$ superimpose almost perfectly with those of Helios at 1 au in the range $350<V<600 \mathrm{~km} \mathrm{~s}^{-1}$. Above $600 \mathrm{~km} \mathrm{~s}^{-1}$ the Wind $T_{e}$ is anticorrelated with $V$, demonstrating that for these highest speeds the pristine electron temperature versus bulk speed anticorrelation is conserved up to one au.

As the final conclusion of our analysis, we show in Figure 8 that this tendency for different electron thermal gradients for different speed regimes is also valid at the radial distances covered by the Ulysses probe. For this reason we have used the electron moments of the Ulysses electron analyzer (Bame et al. 1992). We have restricted our analysis to the period comprised between the launch in 1990 October and the end of the first orbit on 1998 February. We have first divided these data into in-ecliptic regions, with an absolute value of the spacecraft heliolatitude $\lambda$ smaller than $20^{\circ}$, and high-latitude regions, with $|\lambda|>50^{\circ}$. The high-latitude data correspond to the well-known period, during solar minimum, when Ulysses was immersed in a very fast wind emanating from polar coronal holes (McComas et al. 2003). The in-ecliptic electron data are represented by the black triangles, squares, and crosses in Figure 8 , while the high-latitude data are represented by the diamonds. For the in-ecliptic electron data we have applied the same binning technique for speeds and radial distances as previously used. The black triangles in Figure 8 represent the $\left(V_{i}, T_{e i}\right)$ medians for the radial range $1.42<$ au $<1.95$, the black squares are for $3.01<\mathrm{au}<3.54$, and the crosses are for $4.60<\mathrm{au}<5.13$. It is striking to see in this figure how the 

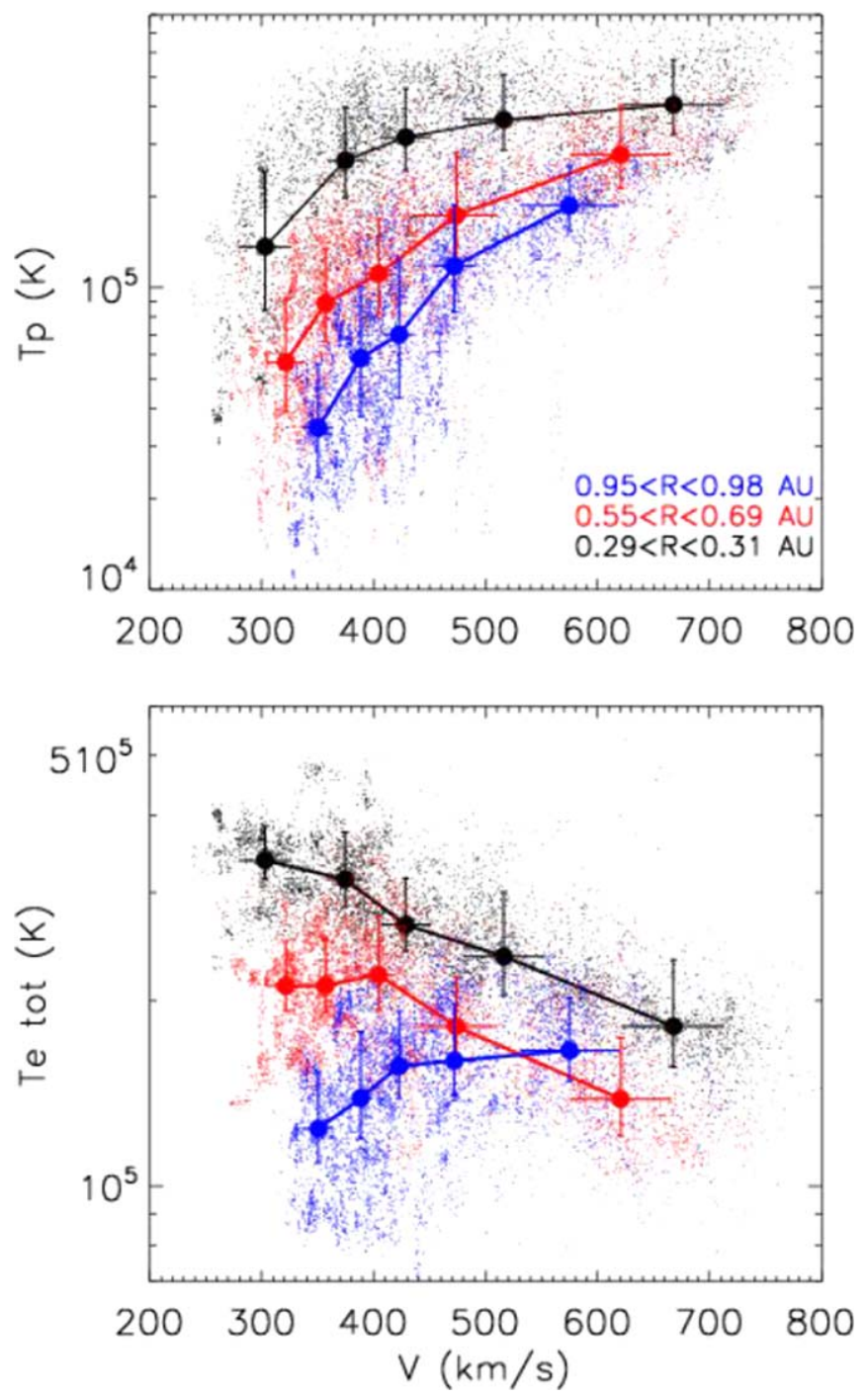

Figure 7. Upper panel: proton temperature as a function of the bulk speed for all the points (light dots) and the medians (thick dots) for the first (black), the sixth (red), and the tenth (blue) radial bins. These three radials bins are indicated in the figure. Lower panel: same as above for the electrons.

Table 1

Linear Pearson Correlation Coefficients for $\left(T_{p}, V\right)$ and $\left(T_{e}, V\right)$ for Three Different Radial Distances

\begin{tabular}{lccc}
\hline \hline & Protons & $\begin{array}{c}\text { Electrons } \\
\text { (Štverák et al. 2009) }\end{array}$ & $\begin{array}{c}\text { Electrons } \\
\text { (Berčič et al. 2019) }\end{array}$ \\
\hline $0.29<R_{\mathrm{AU}}<0.31$ & 0.55 & -0.70 & -0.94 \\
$0.55<R_{\mathrm{AU}}<0.69$ & 0.72 & -0.50 & -0.81 \\
$0.95<R_{\mathrm{AU}}<0.98$ & 0.75 & 0.31 & -0.32 \\
\hline
\end{tabular}

primordial anticorrelation in the near solar corona turns into a rough global correlation between $V$ and $T_{e}$ very far from the Sun. As for the high-latitude data, the radial distance covered is comprised between 1.5 and 3.7 au. When applying our binning technique for these data, and even with different numbers of radial bins, we observe that the $\left(V_{i}, T_{e i}\right)$ medians are all superimposed on each other. We have thus defined four velocity bins for all the high-latitude electron data, independently of the radial distance, and displayed them in Figure 8 with diamonds. As one can see, and contrary to the Ulysses in-

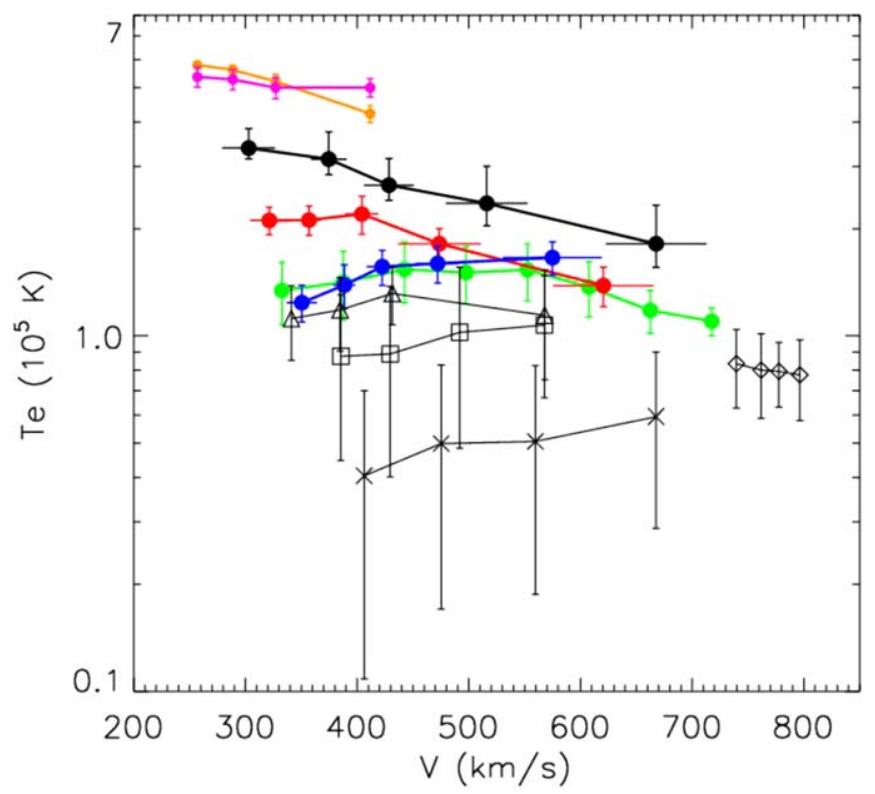

Figure 8. Combined PSP, Helios, and Wind electron temperature variations as a function of the bulk speed. For PSP $T_{e, \mathrm{QTN}}$ is displayed in magenta, while $1.47 \times T_{c, \text { SPAN }}$ is displayed in orange. The black, red, and blue curves are the Helios medians displayed in Figure 7. The green curve corresponds to the medians of the total electron temperatures obtained with the 3DP electrostatic analyzer on board Wind (Lin et al. 1995). The black triangles represent the Ulysses $\left(V_{i}, T_{e i}\right)$ medians for the radial range $1.42<$ au $<1.95$, the black squares are for $3.01<$ au $<3.54$, and the crosses are for $4.60<$ au $<5.13$. The diamonds represent Ulysses $\left(V_{i}, T_{e i}\right)$ medians for the high-latitude regions (see the text for more details).

ecliptic data, $V$ and $T_{e}$ are anti-correlated when the bulk speed is large, even far from the Sun.

\section{Conclusions}

In this paper we have discussed the first measurements of electron temperatures from PSP over its first orbit, with particular emphasis on the first perihelion pass. We have shown that measurements obtained independently using the QTN techniques from the FIELDS experiment are in broad agreement with the direct distribution function results from SPAN on SWEAP. These results show that the nascent, young solar wind observed around 0.15 au displays a strong anticorrelation of wind speed with electron temperature, in agreement with freezing-in temperature results previously obtained by Ulysses and ACE (Geiss et al. 1995; Gloeckler et al. 1998). In addition, the new analysis of the electron temperatures with Helios, Wind, and Ulysses presented here shows how the in situ anticorrelation disappears with increasing distance from the Sun, as the solar wind continues to accelerate, moving outward and perhaps also beginning to mix. More specifically, the radial electron temperature gradients measured with Helios are found to be very different depending on the solar wind speed, with electron temperatures in the slow wind cooling much faster with distance from the Sun than electrons in faster winds. The end result is that the electron temperature wind speed anticorrelation measured in situ by PSP and Helios is lost with increasing radial distances from the Sun. This result raises challenging questions about the heating and cooling mechanisms for electrons occurring in different solar wind stream types, and the possible role of evolving in situ dynamics. While it is agreed that the amplitude of turbulent fluctuations, especially in faster wind 
speeds, is sufficient to provide the required heating of protons and ions in the solar wind, for the electrons the interplay of heating, cooling, and thermal conduction, via electromagnetic field fluctuations, instabilities, and dynamical evolution of the different parts of the distribution function, and Coulomb collisions for the slower parts of the distribution function, are more complex and there is no clear relationship between turbulence and heating. So the questions of why the solar wind is born with an anticorrelation between speed and electron temperature, and why the temperature gradients are so different for different solar wind streams are in need of urgent theoretical exploration.

Parker Solar Probe was designed, built, and is now operated by the Johns Hopkins Applied Physics Laboratory as part of NASA's Living with a Star (LWS) program (contract NNN06AA01C). Support from the LWS management and technical team has played a critical role in the success of the Parker Solar Probe mission. The FIELDS experiment on the Parker Solar Probe spacecraft was designed and developed under NASA contract NNN06AA01C. The first author wishes to thank CNES \& CNRS for their support.

\section{ORCID iDs}

M. Maksimovic (ib https://orcid.org/0000-0001-6172-5062

S. D. Bale (ib https://orcid.org/0000-0002-1989-3596

J. W. Bonnell (iD https://orcid.org/0000-0002-0675-7907

A. W. Case (iD https://orcid.org/0000-0002-3520-4041

T. Dudok de Wit (iD https://orcid.org/0000-0002-4401-0943

K. Goetz (iD https://orcid.org/0000-0003-0420-3633

J. S. Halekas (iD https://orcid.org/0000-0001-5258-6128

P. R. Harvey (iD https://orcid.org/0000-0002-6938-0166

K. Issautier (1) https://orcid.org/0000-0002-2757-101X

J. C. Kasper (iD https://orcid.org/0000-0002-7077-930X

K. E. Korreck (1D https://orcid.org/0000-0001-6095-2490

V. Krishna Jagarlamudi (iD https://orcid.org/0000-00016287-6479

D. E. Larson (1) https://orcid.org/0000-0001-5030-6030

R. Livi (i) https://orcid.org/0000-0002-0396-0547

R. J. MacDowall (iD https://orcid.org/0000-0003-3112-4201

D. M. Malaspina (iD https://orcid.org/0000-0003-1191-1558

M. M. Martinović (i) https://orcid.org/0000-0002-7365-0472

N. Meyer-Vernet (i) https://orcid.org/0000-0001-6449-5274

M. Moncuquet (D) https://orcid.org/0000-0002-9621-0365

M. Pulupa (i) https://orcid.org/0000-0002-1573-7457

C. Salem (iD https://orcid.org/0000-0002-6536-1531

M. L. Stevens (iD https://orcid.org/0000-0002-7728-0085

M. Velli (i) https://orcid.org/0000-0002-2381-3106

P. L. Whittlesey (iD https://orcid.org/0000-0002-7287-5098

\section{References}

Arya, S., \& Freeman, J. W. 1991, JGR, 96, 14183

Bale, S. D., Goetz, K., Harvey, P. R., et al. 2016, SSRv, 204, 49

Bame, S. J., McComas, D. J., Barraclough, B. L., et al. 1992, A\&AS, 92, 237

Berčič, L., Maksimović, M., Landi, S., \& Matteini, L. 2019, MNRAS, 486, 3404
Cane, H. V. 1979, MNRAS, 189, 465

Chateau, Y. F., \& Meyer-Vernet, N. 1989, JGR, 94, 15407

Chateau, Y. F., \& Meyer-Vernet, N. 1991, JGR, 96, 5825

Couturier, P., Hoang, S., Meyer-Vernet, N., \& Steinberg, J. L. 1981, JGR, 86, 11127

Cranmer, S. R. 2002, SSRv, 101, 229

David, C., Gabriel, A. H., Bely-Dubau, F., et al. 1998, A\&A, 336, L90

Doschek, G. A., Feldman, U., Laming, J. M., Schühle, U., \& Wilhelm, K. 2001, ApJ, 546, 559

Feldman, W. C., Asbridge, J. R., Bame, S. J., Montgomery, M. D., \& Gary, S. P. 1975, JGR, 80, 4181

Fox, N. J., Velli, M. C., Bale, S. D., et al. 2016, SSRv, 204, 7

Geiss, J., Gloeckler, G., von Steiger, R., et al. 1995, Sci, 268, 1033

Gloeckler, G., Cain, J., Ipavich, F. M., et al. 1998, SSRv, 86, 497

Gloeckler, G., Zurbuchen, T. H., \& Geiss, J. 2003, JGRA, 108, 1158

Halekas, J. S., Whittlesey, P., Larson, D. E., et al. 2020, ApJS, doi:10.3847/ $1538-4365 / \mathrm{ab} 4 \mathrm{cec}$

Horaites, K., Boldyrev, S., \& Medvedev, M. V. 2019, MNRAS, 484, 2474

Issautier, K., Meyer-Vernet, N., Moncuquet, M., Hoang, S., \& McComas, D. J. 1999, JGR, 104, 6691

Kasper, J. C., Abiad, R., Austin, G., et al. 2016, SSRv, 204, 131

Ko, Y.-K., Fisk, L. A., Geiss, J., Gloeckler, G., \& Guhathakurta, M. 1997, SoPh, 171, 345

Kohl, J. L., Strachan, L., \& Gardner, L. D. 1996, ApJL, 465, L141

Le Chat, G., Issautier, K., Meyer-Vernet, N., \& Hoang, S. 2011, SoPh, 271,141

Lin, R. P., Anderson, K. A., Ashford, S., et al. 1995, SSRv, 71, 125

Lopez, R. E., \& Freeman, J. W. 1986, JGR, 91, 1701

Maksimovic, M., Bougeret, J. L., Perche, C., et al. 1998, GeoRL, 25, 1265

Maksimovic, M., Gary, S. P., \& Skoug, R. M. 2000, JGR, 105, 18337

Maksimovic, M., Hoang, S., Meyer-Vernet, N., et al. 1995, JGR, 100, 19881

Maksimovic, M., Pierrard, V., \& Riley, P. 1997, GeoRL, 24, 1151

Maksimovic, M., Zouganelis, I., Chaufray, J. Y., et al. 2005, JGRA, 110, A09104

Manning, R., \& Dulk, G. A. 2001, A\&A, 372, 663

Marsch, E., Pilipp, W. G., Thieme, K. M., \& Rosenbauer, H. 1989, JGR, 94, 6893

Martinović, M. M., Zaslavsky, A., Maksimović, M., et al. 2016, JGRA, 121,129

Matthaeus, W. H., Elliott, H. A., \& McComas, D. J. 2006, JGRA, 111, A10103

McComas, D. J., Elliott, H. A., Schwadron, N. A., et al. 2003, GeoRL, 30,1517

Meyer-Vernet, N. 1979, JGR, 84, 5373

Meyer-Vernet, N., Issautier, K., \& Moncuquet, M. 2017, JGRA, 122, 7925

Meyer-Vernet, N., \& Perche, C. 1989, JGR, 94, 2405

Moncuquet, M., Meyer-Vernet, N., Issautier, K., et al. 2020, ApJS, doi:10. $3847 / 1538-4365 / a b 5 a 84$

Novaco, J. C., \& Brown, L. W. 1978, ApJS, 221, 114

Parker, E. N. 1958, ApJ, 128, 664

Pilipp, W. G., Miggenrieder, H., Montgomery, M. D., et al. 1987, JGR, 92, 1075

Pulupa, M., Bale, S. D., Badman, S. T., et al. 2020, ApJS, doi:10.3847/1538$4365 / \mathrm{ab} 5 \mathrm{dc0}$

Pulupa, M., Bale, S. D., Bonnell, J. W., et al. 2017, JGRA, 122, 2836

Rosenbauer, H., Schwenn, R., Marsch, E., et al. 1977, JGZG, 42, 561

Schwenn, R., Rosenbauer, H., \& Miggenrieder, H. 1975, RF, 19, 226

Scudder, J. D. 1992a, ApJ, 398, 299

Scudder, J. D. 1992b, ApJ, 398, 319

Štverák, Š., Maksimovic, M., Trávníček, P. M., et al. 2009, JGRA, 114, A05104

Štverák, Š., Trávníček, P. M., \& Hellinger, P. 2015, JGRA, 120, 8177

Totten, T. L., Freeman, J. W., \& Arya, S. 1995, JGR, 100, 13

von Steiger, R., \& Zurbuchen, T. H. 2011, JGRA, 116, A01105

Wilhelm, K., Marsch, E., Dwivedi, B. N., et al. 1998, ApJ, 500, 1023

Zaslavsky, A., Meyer-Vernet, N., Hoang, S., Maksimovic, M., \& Bale, S. D. 2011, RaSc, 46, RS2008

Zouganelis, I. 2008, JGRA, 113, A08111 